\title{
Chronic wrist pain: diagnosis and management. Development and use of a new algorithm
}

\author{
Richard M van Vugt, Johannes W J Bijlsma, Arno C van Vugt
}

\begin{abstract}
Objective-Chronic wrist pain can be difficult to manage and the differential diagnosis is extensive. To provide guidelines for assessment of the painful wrist an algorithm was developed to encourage a structured approach to the diagnosis and management of these patients.

Methods-A review of the literature on causes of chronic wrist pain was undertaken; history taking, physical examination and imaging studies were evaluated systematically to determine which of the many potential conditions was the cause of the wrist pain. Chronic wrist pain was subdivided into pain of probable intraarticular or extra-articular origin. By means of this classification a clinical algorithm was developed to establish a diagnosis and its clinical usefulness was tested in a prospective study of 84 patients presenting to our outpatient clinic.

Results-A definite diagnosis could be established in $59 \%$ (49 of 84 ) of the cases by careful history taking, extensive physical examination, plain radiographs, ultrasound examination and bone scintigraphy. In $19 \%$ of the cases (16 of 84 ) a probable diagnosis was made resulting in a total figure $78 \%$ (65 of 84). Additional imaging studies (arthrography, magnetic resonance imaging and computed tomography) increased the definite diagnoses to $70 \%$ (59 of 84$)$.

Conclusion-The algorithm proved easy to use and by the use of careful history taking, thorough physical examination and simple imaging techniques (ultrasonography and scintigraphy) a diagnosis was made in $78 \%$ of cases.

(Ann Rheum Dis 1999;58:665-674)
\end{abstract}

Department of

Rheumatology and

Clinical Immunology,

F02.127, University

Medical Centre

Utrecht, POBox 85500, 3508 GA Utrecht, the Netherlands

Correspondence to:

Dr R M van Vugt.

Accepted for publication 19 June 1999

Determination of the cause of chronic pain referred in the wrist is frequently a challenge. This is largely because of the anatomical and biomechanical characteristics of the distal radioulnar, radiocarpal and midcarpal joints as well as the complexity of the soft tissue envelope that surrounds them. Within a small area bounded by the distal radius and ulna proximally and the bases of the metacarpals distally, there is a concentration of intimately related structures, each of which can be the site of injury, degeneration or disease and, consequently, the source of pain. ${ }^{12}$ The history and physical examination usually lead to early identification of the cause of hand pain. In some cases, however, the aetiology of the pain is not obvious and a diagnostic problem arises. These patients may go from one doctor to the next without finding an explanation for and/or treatment of their symptoms. Management of this chronic wrist pain in the patient with normal radiographs of the wrist without pathognomonic clinical findings can be frustrating for both the patient and the physician. ${ }^{3}$ As rheumatologists are encountering this problem with increasing frequency, the objective of this article is to make rheumatologists more aware of the diagnosis and treatment of wrist disorders. As the evaluation of a patient with wrist pain requires an extensive medical history, thorough physical examination and knowledge of the imaging studies available, we assessed the successive steps of the examination. In addition literature on the different causes of pain in the wrist was reviewed.

To provide guidelines for assessment of the painful wrist we developed an algorithm (see fig 2) to encourage a structured approach to the diagnosis and management of these patients. By means of a prospective study we tested the clinical usefulness of this algorithm.

\section{Clinical evaluation}

HISTORY

The first step in taking a patient's history is to listen carefully to their complaints, bearing in mind that most patients with chronic wrist pain have already visited other physicians and may have a preconceived notion of what the physician wants to hear. ${ }^{1}$ The patient should be encouraged to describe the location of the pain, how long it has persisted, its intensity, and any activity or wrist motion that aggravates or relieves the pain. Frequently, patients use terms to describe the quality of their pain and tenderness that indicate superficial involvement (suggestive of tendinitis) or deep involvement (suggestive of bone, ligament, or joint involvement). Moreover, the patient's description of pain may suggest paresthesia rather than underlying point tenderness.

Any traumatic event should be described carefully, in an attempt to determine the exact 
mechanism of injury. Questions in the history taking should include past employment, even when the patient does not consider the pain to be work related. For example, patients exposed to vibrating instruments, such as a jackhammer, or those who use the hand and wrist repeatedly and in the same manner during a day's work may develop wrist pain that is directly related to on the job tasks. Overuse syndromes, frequently encountered in competitive athletes or musicians, can produce soft tissue inflammation and/or attrition. Knowledge of the effect of wrist pain on the patient's daily activities, including leisure activities, is essential for therapeutic management.

In addition, a complete medical history, with special attention to metabolic diseases, skin diseases, surgery, and other instances of joint involvement (in the past) must be obtained. A thorough review of the body systems, including all notable systemic illnesses, may reveal additional relevant information. ${ }^{4} 6$

\section{PHYSICAL EXAMINATION}

The physical examination should not be restricted to the wrist; examination of the entire upper extremity including the cervical spine is essential. Neck problems, for example, may play an important part in referred pain in the wrist. Other joints or areas of symptomatology should also be explored. The wrist examination begins with a careful inspection for specific areas of swelling, erythema, warmth, nodules, skin lesions, and obvious deformities or prior surgical incisions. Tenderness is localised to a specific anatomic structure, if possible. In most cases, the tender area can be isolated, enabling the examiner to continue the examination with special manoeuvres (Grind test for osteoarthritis CMC1, Finkelstein's test for Quervain's tendosynovitis, Watson's test for scaphoid pathology, Allen's test for vasculopathy). Active and passive ranges of motion are recorded and the search for "clicks" and crepitus should be diligent.

A complete neurovascular examination is performed. When the patient is evaluated for carpal tunnel syndrome, the Tinel and Phalen tests should be performed. The physical examination of patients with chronic wrist pain should also include separate testing of each muscle-tendon unit crossing the wrist.

In some cases, a thorough history and a complete and meticulously performed physical examination will result in a clear diagnosis. If not, a suspected diagnosis can almost always be categorised as intra-articular or extra-articular, with further evaluations planned to pinpoint the diagnosis.

RADIOGRAPHIC EXAMINATION

After history taking and the physical examination, the conventional radiographic study is the next step in the evaluation of wrist disease. Unless otherwise indicated by clinical findings, the initial radiographic examination should consist of three views. ${ }^{7}$

Standard radiographs include the posteroanterior (PA), oblique (PA oblique or AP oblique) and lateral views. The findings of the patient's history and physical examination will dictate the oblique views needed for evaluation of the radial (PA oblique) or ulnar part (AP oblique) of the wrist. The conventional radiographs are examined for bony abnormalities (fractures, cortical interruption, degree and pattern of mineralisation) and the width and symmetry of joint spaces. The ligamentous architecture is assessed by determining whether the three carpal arcs of the wrist and parallelism of the joints are maintained. ${ }^{8}$ The arcs join the proximal and distal articular surfaces of the scaphoid, lunate, and triquetrum and the proximal articular surface of the capitate and hamate. A broken arc usually indicates disruption of joint integrity at that site. The PA radiograph should also include the entire length of the third metacarpal because this allows later measurement of carpal height and carpal-ulnar distance ratios, if needed. The lateral view is extremely important for evaluation of radiolunocapitate aligmnent and assessment of radioscaphoid, lunoscaphoid, and capitoscaphoid relations. In the normal wrist, the radius, lunate, and capitate are collinear. In case of "zigzag" alignment of the radiolunocapitate link, carpal instability must be suspected. ${ }^{9}$ If the long axis of the capitate is aligned palmar or dorsal (because of volar or dorsal rotation of the "intercalated segment" or the lunate) to the radius, the terms VISI (volar intercalated segment instability) or DISI (dorsal intercalated segment instability) are used, respectively.

Supplemental views of the wrist should be dictated by the findings of the clinical examination, such as the carpal tunnel view to evaluate the bony tubercles and soft tissue structures of the carpal tunnel, "clenched-fist" radiographs for enhancing detection of scapholunate dissociations and spot films or tangential films of the painful region for patients with pain isolated at one site. ${ }^{8}$

When routine radiographs reveal abnormalities, they are often diagnostic, especially for patients with osteoarthritic problems and fractures. If the findings on these radiographs are normal, additional evaluation should be guided by the findings of the physical examination. A seeming myriad of imaging studies is available, including scintigraphy, ultrasonography, arthrography, magnetic resonance imaging, and computed tomography. When clinical examination suggests superficial involvement and extra-articular pathology is suspected, an ultrasound examination should be the next step.

Musculoskeletal ultrasound is a quick and easy method of obtaining diagnostic information from dynamic studies and bilateral comparisons with low patient discomfort. It not only demonstrates abnormalities but also provides the opportunity to compress that region accurately to see if this reproduces or accentuates the patient's characteristic symptoms - that is, ultrasonography can be considered an extension of the physical examination. Recent improvements in resolution and sensitivity have led to better understanding and definition of anatomical structures and disease states as well as new applications, such as the 
ultrasound guided synovial biopsy or injection therapy. ${ }^{10}$ If intra-articular pathology is suspected or the findings on ultrasound examination are normal, a bone scan should be made. Although bone scans are non-specific, they yield information that helps the physician plan additional examinations and tests. ${ }^{1} \mathrm{~A}$ three phase study consists of an "arteriographic" phase, a "blood pool/soft tissue" phase, and a "bone" or delayed phase. However, some acute and subacute soft tissue injuries, such as ligamentous damage, can cause mildly to moderately increased uptake in both bones spanned by the ligament. ${ }^{11}$ For patients with abnormal findings on the bone scan spot films, computed tomography (CT) or magnetic resonance imaging (MRI) is recommended. If the bone scan is negative, the physicians's options are to stop further investigations (based on clinical impression), consider an arthrogram or a diagnostic arthroscopy. In some instances, a negative bone scan may reinforce an impression of malingering. If the history and physical examination (clicking or snapping) suggest that the patient's problems stem from ligamentous injuries or injuries of the triangular fibrocartilage complex, cineradiography (an active motion study for picking up instability patterns) or an (three compartment) arthrogram under fluoroscopic control must be done. In cineradiography the wrist is moved through a full range of motion, with specific attempts to recreate the stresses and positions known by the patient to cause the click or pop. $^{2}{ }^{12}$ Subsequent examination is arthrography, which serves to establish the integrity of the capsular structures and intrasynovial interosseous ligaments, especially the scapholunate and lunotriquetral ligaments and the triangular fibrocartilage. ${ }^{13}$ It may also show abnormal infolding of the synovium or the corrugated appearance consistent with localised synovitis. Arthrograms are diagnostic when they show an abnormal leak of opaque material between the radiocarpal and midcarpal or distal radioulnar spaces. To be sure of the diagnosis, the flow of dye across these articulations is viewed directly by fluoroscopy. This finding must be evaluated carefully, however, in relation to the patient's age, complaints, and clinical findings. Communication between the different compartments of the wrist is not necessarily the result of trauma or disease. ${ }^{7}$ The cineradiographic study may be combined with arthrography for greater efficiency and convenience. Often, abnormalities found on the arthrogram enable the examiner to pinpoint a diagnosis.

Good quality $M R I$ can occasionally visualise the ligamentous and cartilaginous structures of the wrist, particularly the triangular fibrocartilage complex, and can reveal the presence or absence of occult ganglions and tendinitis. ${ }^{14} 15$ As MRI has only proved useful in a limited number of pathological conditions ultrasonography and radionuclide imaging are generally performed first because they are much less expensive and more readily available.

Finally arthroscopy of the wrist can be used for assessment of the intra-articular pathology (especially ligaments and the articular cartilage) of the radiocarpal, mediocarpal and distal radioulnar joints. It is one of the more useful tools available to the physician, being both diagnostic and therapeutic. Arthroscopy causes minimal invasion, hence patients rehabilitate quickly with fewer complications. ${ }^{16} 17$

\section{Differential diagnosis}

Although most causes of chronic wrist pain require further evaluation, some can readily be diagnosed during routine history taking and physical examination, for example, distal radioulnar joint and extensor carpi ulnaris tendon subluxation or evident manifestations of inflammatory arthropathy (tables 1 and 2).

A fall onto an outstretched hand with subsequent tenderness in the anatomical snuff box is the classic mechanism of a scaphoid fracture.

The scaphoid fracture is one of the most common carpal fractures in the wrist. Several factors contribute to the scaphoid's deserved reputation as a source of frequent complications.

The scaphoid is difficult to visualise on radiographs: regardless of the view scaphoid fractures are invisible in as many as $20 \%$ of the cases. In addition, because of mobility, unstable fractures tend to angulate dorsally, which leads to an increased incidence of malunion, carpal instability and avascular necrosis. ${ }^{18}$

Fractures of the other carpal bones are less common. They can remain unnoticed for some time and require special views for visualisation, for instance a carpal tunnel view for fracture of the hook of the hamate. ${ }^{7}$

The ligaments are intended to ensure carpal stability. ${ }^{2}$ Carpal instability, which can be secondary to attenuation or a partial or complete rupture, can be static or dynamic.

Table 1 Differential diagnosis of chronic wrist pain

\begin{tabular}{l}
\hline Intra-capsular \\
Fracture \\
Ligament tear \\
static instability pattern \\
dynamic instability pattern \\
triangular fibrocartilage complex tear \\
Distal radioulnar joint subluxation \\
Arthritis \\
rheumatoid arthritis \\
other connective tissue diseases \\
psoriasis \\
metabolic diseases (gout/pseudogout/ \\
hyperparathyreoidism) \\
infectious diseases (common/atypical agent) \\
Osteoarthritis \\
primary (CMC 1, STT joint) \\
secondary (SLAC) \\
Neoplasm \\
enchondroma \\
osteoid osteoma \\
pigmented villonodular synovitis \\
other (giant cell tumour) \\
Ganglia (extra-osseous/intra-osseous/occult) \\
Avascular necrosis \\
lunate (Kienbock's) \\
scaphoid (Preiser's) \\
Other (osteochondromatosis/carpal boss/carpal coalition) \\
Extra-capsular \\
Neuropathy \\
median nerve (carpal tunnel) \\
ulnar nerve (Guyon's canal) \\
distal posterior interosseous nerve syndrome \\
thoracic outlet compression syndrome \\
neuroma \\
Tendinopathy \\
extensor carpi ulnaris tendon subluxation \\
tendovaginitis (De Quervain's) \\
repetitive strain injury \\
multisystemic disease \\
infectious \\
\end{tabular}


Table 2 Differential diagnosis of chronic wrist pain with most discriminating features in the history, examination and imaging for each diagnosis

\begin{tabular}{|c|c|c|c|}
\hline Diagnosis & History & Examination & Imaging \\
\hline \multicolumn{4}{|l|}{ Intra-capsular } \\
\hline Fracture & traumatic event & point tenderness & SR, SV (carpal tunnel) or CT \\
\hline \multirow[t]{3}{*}{ Ligament tear } & \multirow[t]{3}{*}{ clicking, (unnoticed) traumatic event } & point tenderness & SR: malalignement (lateral "zigzag") or dissociation \\
\hline & & ST: Watson's test (scaphoid), & carpal bones \\
\hline & & McMurray's test (TFCC) & AG: leakage between different compartments of wrist \\
\hline \multirow[t]{2}{*}{ DRUJ subluxation } & \multirow[t]{2}{*}{ painful pronation and supination } & prominent ulnar head & SR: lateral view unreliable \\
\hline & & $\begin{array}{l}\text { ST: DRUJ compression during pro- and } \\
\text { supination }\end{array}$ & CT: axial view \\
\hline \multirow[t]{2}{*}{ Arthritis } & \multirow{2}{*}{$\begin{array}{l}\text { spontaneous swelling, } \\
\text { symptomatology other joints or } \\
\text { systemic complaints }\end{array}$} & \multirow[t]{2}{*}{$\begin{array}{l}\text { swelling, warmth, erythema, decreased painful } \\
\text { range of movement }\end{array}$} & $\begin{array}{l}\text { SV: decreased mineralisation, cortical interruption or } \\
\text { crystal deposits }\end{array}$ \\
\hline & & & $\begin{array}{l}\text { US: hydrops or synovial proliferation } \\
\text { US punction/biopsy }\end{array}$ \\
\hline Osteoarthritis & $\begin{array}{l}\text { (gradually) progressive pain and } \\
\text { stiffness }\end{array}$ & $\begin{array}{l}\text { decreased passive range of movement, crepitus } \\
\text { ST: (pisiform) Grind test }\end{array}$ & $\begin{array}{l}\text { SR: with PA oblique (CMC-I, STT) or AP oblique } \\
\text { (pisotriquetral) }\end{array}$ \\
\hline Neoplasm & & ND & SR \\
\hline (osteoid osteoma) & (pain at night, relief of salicylates) & & $\begin{array}{l}\text { CT/MRI: determining extent lesion and effect on } \\
\text { surrounding neurovascular structures } \\
\text { (SR/CT: nidus with radiolucent or sclerotic centre) }\end{array}$ \\
\hline \multirow{2}{*}{ Ganglia } & \multirow{2}{*}{ localised painful swelling } & \multirow[t]{2}{*}{ tense discrete subcutaneous swelling } & US: anechoic cyst area \\
\hline & & & MRI: occult or intraosseus \\
\hline \multirow[t]{2}{*}{ Avascular necrosis } & \multirow[t]{2}{*}{ pain without swelling } & \multirow[t]{2}{*}{ decreased painful range of movement } & SR: subtly increased radiodensity \\
\hline & & & BS: increased uptake \\
\hline Other & ND & ND & \\
\hline (carpal boss) & (swelling base 2 nd or 3th metacarpal) & (tender bony prominence) & (SV: carpal boss view) \\
\hline \multicolumn{4}{|c|}{ (9) } \\
\hline \multirow[t]{4}{*}{ Neuropathy } & \multirow{4}{*}{$\begin{array}{l}\text { paresthesia, nocturnal excacerbation } \\
\text { of symptoms, metabolic disease }\end{array}$} & \multirow{4}{*}{$\begin{array}{l}\text { ST: Tinel and Phalen tests, diagnostic lidocaine } \\
\text { injection (neuroma) }\end{array}$} & SR: PA view \\
\hline & & & SV: carpal tunnel view \\
\hline & & & EMG: abnormal nerve conduction time \\
\hline & & & $\begin{array}{l}\text { US: swelling/flattening median nerve and increased } \\
\text { palmar bowing retinaculum }\end{array}$ \\
\hline \multirow[t]{2}{*}{ Tendinopathy } & \multirow{2}{*}{$\begin{array}{l}\text { burning pain, highly repetitious and } \\
\text { forceful motions }\end{array}$} & swelling, crepitus, snapping, & SR: calcifications \\
\hline & & $\begin{array}{l}\text { ST: isometric function tests, forced stretching } \\
\text { (Finkelstein's test) }\end{array}$ & US: thickened tendon with peritendinous effusions \\
\hline
\end{tabular}

(ST: stress test, ND: non-discriminating, SR: standard radiograph, SV: supplemental view, EMG: electromyelogram, AG: arthrography, US; ultrasonography, BS: bone scan, MRI: magnetic resonance imaging).

The scapholunate dissociation is the most common form of carpal instability. It is secondary to the loss of support of both the ligamentum radioscapholunatum and the interosseous scapholunate ligament leading to scapholunate diastasis, palmar flexion of the scaphoid and dorsal flexion of the lunate. This condition is present when the scaphoid is foreshortened on the PA view (cortical ring sign) and the scapholunate interval exceeds $3 \mathrm{~mm}$ (fig 1). On the lateral roentgenogram, the scapholunate angle usually exceeds $60^{\circ}$ and the axis of the capitate is dorsal to that of the radius (DISI)..$^{28}$

The opposite pattern of VISI is seen in triquetrolunate dissociation. Because of the loss of ligamentous constraint the lunate comes under the now uncontested influence of the palmar- flexing scaphoid. Both scaphoid and lunate appear palmar flexed on PA radiographs, the scaphoid is foreshortened (ring sign) and the lunate is triangular in shape, coexisting with a dorsiflexed triquetrum in a "low" or distal position in relation to the lunate, leading to interruption of the proximal arc. This type of instability is more frequent in the rheumatoid wrist. Dynamic forms of carpal instability can only be recognised during cineradiographic studies. ${ }^{2} 8$

Patients with lesions of the triangular fibrocartilage complex complain of a painful click during wrist motions and tenderness is clearly localised to the dorsal anatomic depression, which is immediately distal to the ulnar head. Passive manipulation of the carpal condyle against the head of the ulna, with the wrist in ulnar deviation, will frequently elicit a painful crepitus or roughness or, more rarely, an actual snap (McMurray's test for the wrist). Routine radiographs are negative but valuable nevertheless, for it is important to determine whether an ulna-plus variance coexists with the triangular fibrocartilage tear (ulnocarpal

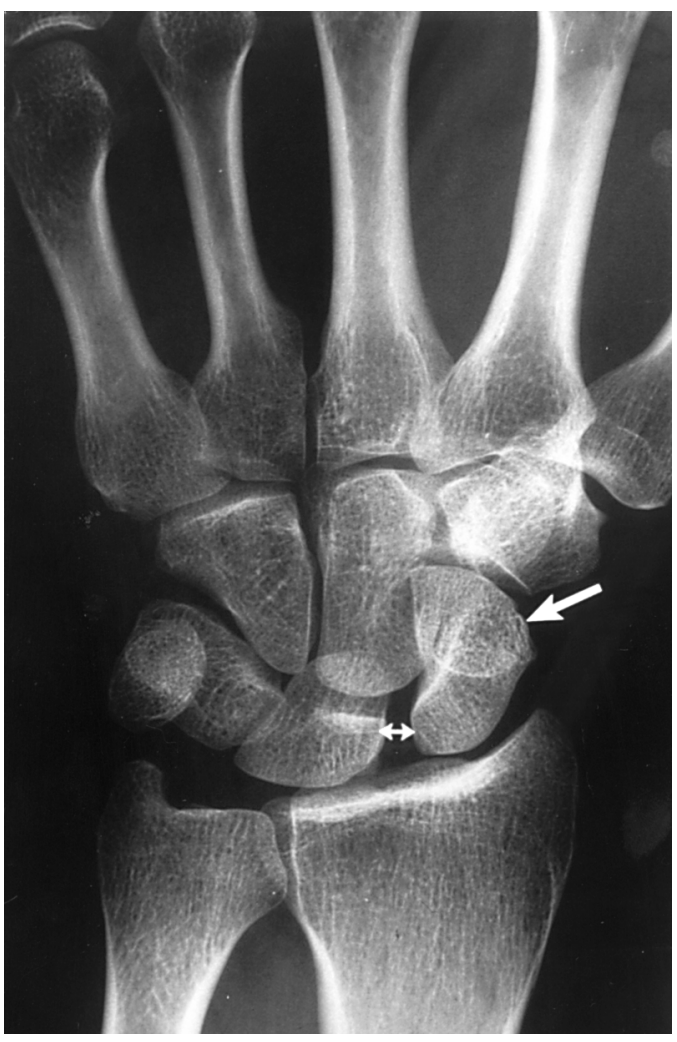

Figure 1 Scapholunate dissociation with scapholunate diastasis, "ring sign" (arrow) and foreshortened appearance of the scaphoid. 
impingement syndrome). This condition is diagnosed on radiographs showing cystic or erosive changes in the ulnar head and along the proximal contour of the lunate. ${ }^{7}$ Although MRI appears promising for detection of defects in the triangular fibrocartilage and intercarpal ligaments, such ultra high resolution imaging requires special coils, software, and technical expertise that are not available at most imaging centres. ${ }^{15}$ Arthrography is a sensitive modality for detection of TFCC tears but arthroscopy offers true evidence of the exact site of the lesion and information about the intraarticular associated causes of ulnar wrist pain such as chondromalacia or synovitis. An added benefit is that many of the pathologies seen can be treated using arthroscopic techniques. ${ }^{192}$ The finding must be carefully evaluated, however, in relation to the patient's age, complaints, and clinical findings. While central perforations are likely to be degenerative and begin after the third decade, peripheral (next to the ulnar styloid) tears are more typical of traumatic avulsions ${ }^{7}$ ).

Chondromalacia of the ulnar head is usually seen in young patients after a fall on the dorsiflexed wrist with the impact predominantly hypothenar or repeated episodes of stressful pronation and supination (work or leisure activity). The pain is localised to the dorsal distal radioulnar area and manipulation of the ulnar head can elict crepitation or a painful snap. ${ }^{7}$ Monarthritis of the wrist is sometimes difficult to establish. ${ }^{6}$ Careful palpation and inspection will usually reveal synovitis but the diagnosis may sometimes be difficult especially when obese patients are examined or the swelling is mild and bilateral. Synovitis, effusion and changes in the capsule are easily detected by ultrasound ${ }^{21}$ with good differentiation between thickening of the synovium itself and a fluid effusion. In selected cases of unclassified joint disease, it may be useful to obtain synovial tissue samples for pathological and bacteriological examination ${ }^{6}$; an ultrasound guided biopsy should be taken. The wrist is frequently affected by rheumatoid arthritis (RA) and is often the first site of the disease. The earliest changes of RA appear on the radiograph as marginal erosions where the synovium is reflected, for example, at the ulnar styloid. ${ }^{22}$ Later in the disease process loss of bone and joint stability produces the typical rheumatoid deformity (carpal collapse, ulnar translocation, carpal supination and radial deviation) of the wrist and hand. Other (multisystemic) connective tissue diseases (systemic lupus erythematosus, sclerodermia) can also present in the initial phase with arthralgia and arthritis. Pain and stiffness are more common than objective abnormalities, although transient joint swelling and effusions can occur. ${ }^{6}$ Psoriatic arthritis can affect any of the joints, including the wrists. Psoriatic arthritis, except for arthritis mutilans, tends to cause less pain and disability than RA; destruction and stiffness are the characteristic findings rather than the typical instability seen in RA. Furthermore, spontaneous wrist fusion is common. ${ }^{23}$

Gouty arthritis and pseudogout are metabolic joint diseases caused by deposition of sodium urate or calcium pyrophosphate crystals in the joint, leading to arthritis. In pseudogout the wrists are the second most commonly affected joint after the knees. Radiographs demonstrate crystal deposits in articular fibrocartilage of the wrist. ${ }^{24}$ Septic arthritis of the wrist can cause destruction of joint cartilage and bony structures. Generally, the diagnosis of acute infection is not problematic, but differentiation between pure soft tissue infection and infection involving the bony structures can be complicated. Furthermore, identification of a chronic infection as the cause of chronic wrist pain may be difficult. ${ }^{25}{ }^{26}$ If there is a clinical suspicion an (ultrasound guided) needle aspiration or synovial biopsy should be taken. The new generation of ultrasonography has proved to be a valuable technique, with a high success rate, for obtaining synovial fluid or membrane samples for pathological and bacteriological examinations. ${ }^{10}$

Osteoarthritis is the most common joint disease. This condition can be primary or secondary, depending on the presence of a preexisting condition. While primary osteoarthritis commonly involves the trapeziometacarpal (CMC-I) or sometimes the trapezioscaphoid (STT) joint, it is uncommon in other parts of the joint. Secondary osteoarthritis of the wrist attributable to an old trauma or infection is very common. In case of malalignment of the scaphoid, degenerative arthritis will progress according to a very specific pattern that leads to an SLAC (scapho-lunate advanced collapse) wrist. Degeneration occurs between the radius and the scaphoid and then between the lunate and capitate. The radiolunate joint is almost never involved. Finally, a scapholunate diastasis develops and the capitate slides in between the lunate and scaphoid. ${ }^{27}$

Neoplasms of the carpal bones themselves are extremely unusual. Enchondromas are occasionally seen in the metacarpals as giant cell tumours have a preference for the distal radius. ${ }^{28}$

Osteoid osteoma is a benign osteoblastic lesion characterised by a nidus that may either be purely radiolucent or have a sclerotic centre. It is often surrounded by a zone of reactive bone formation. The most important clinical symptom is pain that is severest at night and is relieved dramatically by salicylates. Most radiolucent carpal lesions are carpal cysts that have no clinical consequences. ${ }^{1}$

Ganglia are thin walled cysts containing mucoid hyaluronic acid that develop over a joint capsule or tendon sheath. They present as a tense discrete subcutaneous swelling and may be single or multiple. An unusual form is the intraosseous ganglion that appears on radiographs as a radiolucent area in the carpal bones. They may occur spontaneously or in association with such systemic diseases as arthritis. There is often an association with trauma. ${ }^{29} 30$

Osteonecrosis of the carpal bones, which is uncommon, usually involves the proximal scaphoid after a fracture. "Idiopathic" osteonecrosis develops most commonly in the lunate, the so called lunate malacia or Kienbock's disease (or Preiser in case of scaphoid malacia). Plain radiographs may show only a 


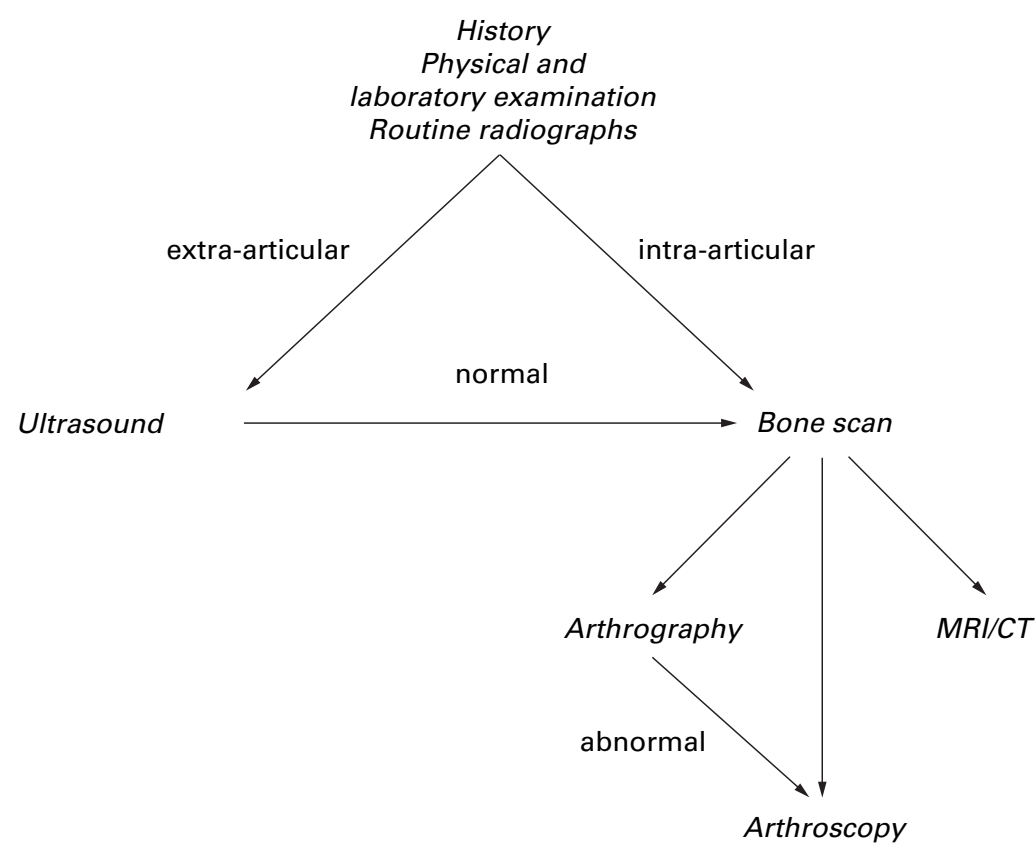

Figure 2 Algorithm for chronic wrist pain.
Overuse syndromes are a common cause of tendinitis, particularly in the "weekend warrior". As a rule the tendons of the musculus abductor pollicis longus and musculus extensor pollicis brevis (De Quervain's) are involved. Pain is experienced on the radial side of the wrist, sometimes with local swelling along the radial tendons. A positive Finkelstein's test (pain over the radial styloid when the patient's thumb is grasped and the hand abducted ulnarward ${ }^{35}$ is the most pathognomonic sign. There are, however, some uncommon locations and types of tendinitis. The flexor carpi radialis tunnel syndrome is an often unrecognised type of tendinitis, as is the flexor carpi ulnaris syndrome. Both syndromes occasionally present with calcification on roentgenograms. ${ }^{26} 36$ There has been a marked increase in reports of the so called repetitive strain injury of the upper extremity. One of the difficulties in evaluating this disorder is the establishment of a diagnosis in the absence of objective physical findings or confirmatory diagnostic images or laboratory data. These issues become more complicated when insurers and attorneys ask physicians to establish a causal relation between the job and the complaints. ${ }^{56}$ Tenosynovitis is frequently seen in inflammatory rheumatic diseases, diabetes mellitus or hypothyroid conditions. Ultrasound examination appears to be a useful method for evaluation as it reveals involvement of the sheaths and tendons in the simplest, most complete and least expensive manner. If a non-infected tenosynovitis is likely, corticosteroid injections can easily be administered under ultrasound guidance.

As physicians, our primary responsibility is to rule out an anatomic lesion before establishing a non-organic cause of the patient's pain. Many patients who are classified as such may have an organic component to their pain, but the overlying non-medical issues confuse the picture and make it impossible to establish a definitive diagnosis. Some patients have unreasonable subjective symptoms and on physical examination, the findings can include a wide gamut of possibilities. These patients have the chronic pain syndrome and should be told they are no longer eligible for medical treatment. In less severe cases psychological consultation, behavioural-based treatment, and employee assistance programmes can be helpful. ${ }^{5}$

\section{Clinical algorithm}

Based on the aspects of history, physical examination and imaging techniques described above and in line with the range of differential diagnoses we developed a practical algorithm (fig 2) for our patients with chronic wrist pain. In the following section our experience with this algorithm will be described.

\section{METHODS}

Between June 1993 and May 1996, 84 patients with chronic wrist pain were referred to our clinic. In accordance with our suggested algorithm a careful history of the wrist condition was recorded, especially location, duration, intensity, aggravating or relieving factors, and the effects of various therapeutic modalities already applied. History taking distal posterior interosseous syndrome. ${ }^{34}$ Diabetes, with its associated neuropathies or cheiroarthropathy, may be an underlying cause of chronic wrist pain. 
Table 3 Characteristics of patients with chronic wrist pain

\begin{tabular}{ll}
\hline Patient characteristics & $\begin{array}{l}\text { Number of patients } \\
(n=84)\end{array}$ \\
\hline History & 21 men / 63 women \\
Sex & mean 32.4 (16-78) \\
Age (y) & $\mathrm{n}=67$ \\
Dominant hand affected & $\mathrm{n}=20$ \\
Pain only during activity & $\mathrm{n}=12$ \\
Paresthesia & $\mathrm{n}=24$ \\
Burning pain & $\mathrm{n}=8$ \\
History of trauma & $\mathrm{n}=48$ \\
History of swelling & $\mathrm{n}=6$ \\
Investigation & $\mathrm{n}=1$ \\
ESR increased & $\mathrm{n}=1$ \\
ANA positivity & $\mathrm{n}=11$ \\
Rheumatoid factor & $\mathrm{n}=49$ \\
Examination & $\mathrm{n}=22$ \\
Swelling & $\mathrm{n}=34$ \\
Tenderness & $\mathrm{n}=20$ \\
Restricted range of movement & $\mathrm{n}=10$ \\
Isometric function tests & \\
Painful forced radial or ulnar deviation \\
Clicking
\end{tabular}

included any past trauma, work and leisure activities. All patients underwent routine radiography and standard laboratory tests (erythrocyte sedimentation rate, complete blood count, thyroid profile and, if clinically indicated, rheumatoid factor, antinuclear antibodies, vitamin $\mathrm{B} 12$, folate and uric acid levels). If the routine radiological examination was normal the patients were separated into two categories on the basis of the findings of history and physical examination: probable intra-articular pathology and probable extra-articular pathology. If extra-articular pathology was suspected a high resolution ultrasound examination was done next. If pathology was observed, depending on the findings, subsequent ultrasound guided puncture, biopsy or corticosteroid injection was carried out. In case of complaints of paresthesia, an electromyelogram and additional radiographic studies of the carpal tunnel or cervical spine were performed. If the results

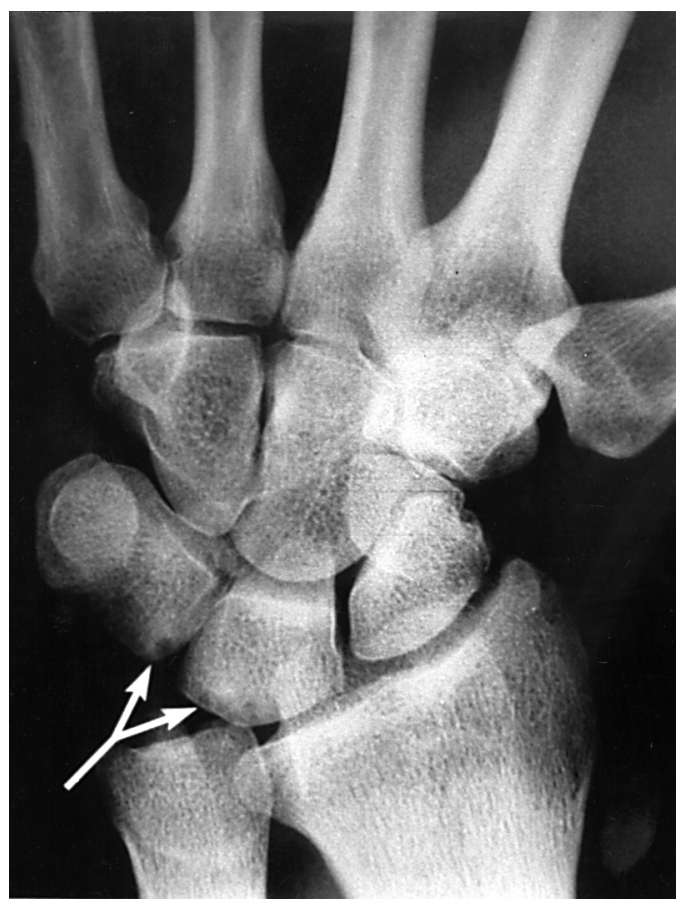

Figure 3 Ulnocarpal impingement syndrome with ulna-plus variance and cystic changes along the proximal contour of the lunate and triquetrum (arrows). were negative the choice was either to proceed with conservative treatment or to continue the examination as for intra-articular pathology.

When intra-articular pathology was suspected a bone scan was performed. If the bone scan was positive, further radiographic studies (MRI or CT) focused on the "hot spot". In case of diffuse involvement synovitis was suspected and an ultrasound guided biopsy was taken. If the bone scan was negative, the choice was to stop further investigation (based on the clinical impression) or, especially in the case of "clicking" or "popping", to perform an arthrogram under fluoroscopic control or arthroscopy. Arthroscopy was performed through the standard three/four radiocarpal portal and the central mid-carpal portal on an outpatient basis.

\section{RESULTS}

Eighty four patients, 21 men and 63 women, 16 to 78 years (mean 32.4 ) were seen (table 3 ). The duration of symptoms ranged from 2 to 72 months, with an average of 13.4 months. The dominant hand was affected in 67 cases, reflecting a predominance of dominant arm problems. The pain was described as nagging without interruption by 40 patients, burning by 24 patients; 12 patients also reported paresthesia. Twenty patients had symptoms only during periods of activity such as work, sports, or playing a musical instrument; however during periods of rest, they were still aware that the wrist was different from the contralateral wrist, even though it was not acutely painful. Eight patients related symptom onset to a possible traumatic event, usually forced twisting. Forty five patients had a past history of a bump or swelling on the dorsum of the wrist.

At examination subtle swelling was present on the dorsoulnar side in four cases, on the dorsoradial side in five cases and the volar side in two cases. Tenderness to palpation without swelling was evident in 49 patients. In 22 patients wrist motion was slightly restricted by pain at the extremes of either flexion/extension or radial and ulnar deviation. Isometric function tests were painful in 34 patients and forced radial or ulnar deviation increased pain in 20 patients. "Clicking" of the wrist was observed in 10 patients.

Laboratory results did not reveal any abnormalities apart from an increased erythrocyte sedimentation rate in six patients, a positive rheumatoid factor in three patients, and a positive antinuclear antibody (ANA 1:400) in another patient.

Thirty three patients who had been seen before by other specialists had normal plain radiographs, 25 of them had already been treated with splinting, cortisone injections, or anti-inflammatory drugs, which did not seem to change the eventual course of the disease in any of the cases. Routine radiographs were diagnostic for nine patients. Osteoarthritis (among others, of the scaphotrapeziotrapezoidal or STT joint) was found in four cases, scapholunate dissociation in one (fig 1), chondrocalcinosis in two, ulnocarpal impingement in one (fig 3) and Kienbock's disease in another patient. 


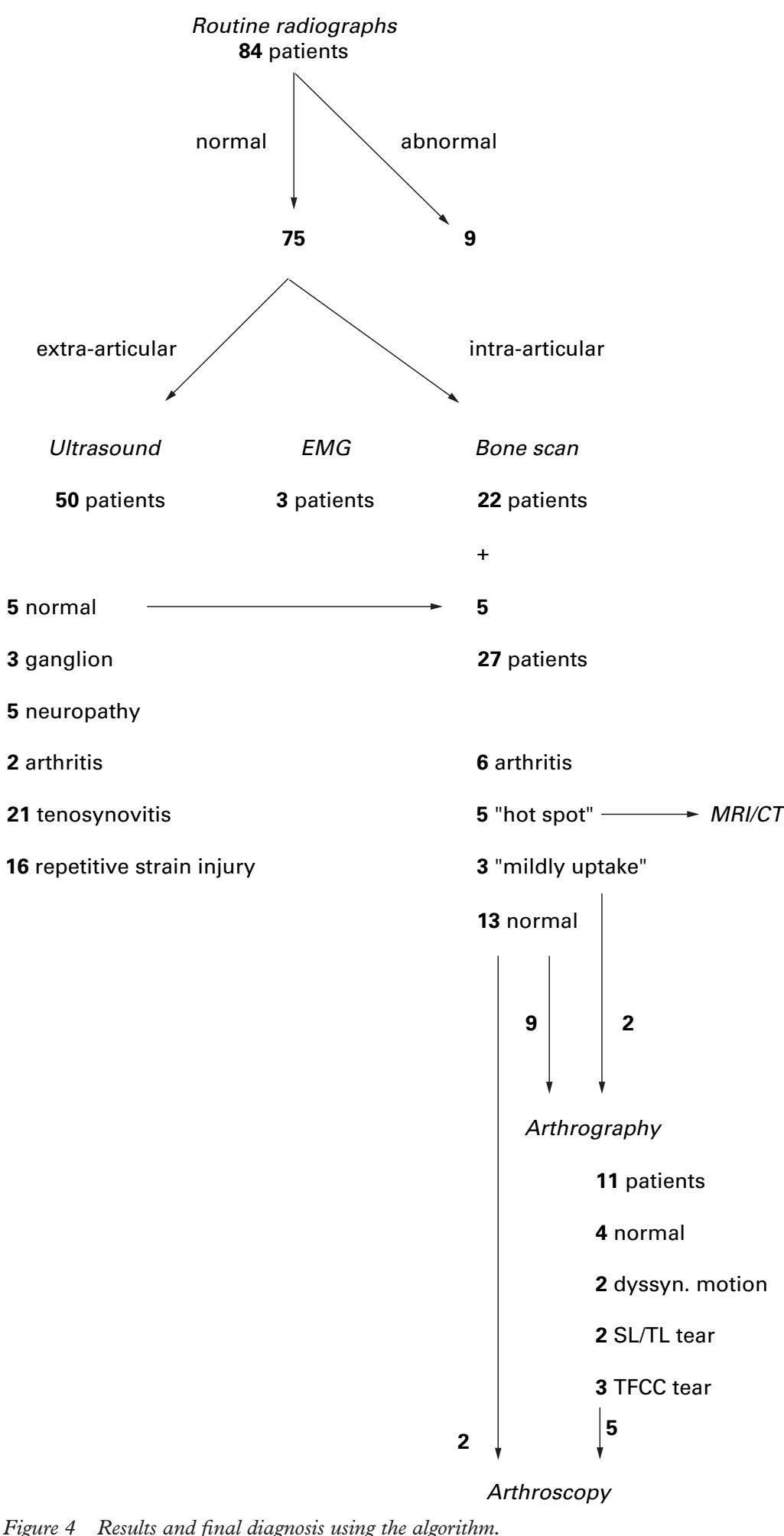

Figure 4 Results and final diagnosis using the algorithm.

Of the remaining patients with normal standard hand radiographs, 53 were categorised as having probable extra-articular pathology and 22 as having probable intra-articular pathology. For four of the 53 patients carpal tunnel syndrome was suspected; electromyelograms confirmed this diagnosis in three cases. The rest of this group (50 patients) with probable extra-capsular pathology underwent ultrasound examination. The diagnoses obtained are shown in figure 4.

Tenosynovitis or tendinitis was observed in 21 patients. In these cases the thickened tendon could be seen embedded in hypoechoic thickening of the tendon sheath or an anechoic peritendinous effusion. One patient had tenosynovitis attributable to an until then unnoticed foreign body. Echo guided surgical exploration showed a thorn in granulomatous tissue (fig 5). Another patient, a tropical fish merchant, had tenosynovitis attributable to infection with myco-bacterium marinum. For 16 patients a non-infected tenosynovitis was considered probable and an ultrasound guided injection of corticosteroids mixed with lidocaine was given. The corticosteroid crystals could be seen spreading diffusely around the tendon. Thirteen of the 16 patients were cured at the follow up clinical examination. Two patients showed intra-articular synovial proliferation. Three patients had an anechoic cystic area compatible with ganglia; two were successfully aspirated and corticosteroids were injected. One required surgical excision.

In three cases the images were compatible with neuropathy: one had a neuroma of the median nerve and two the carpal tunnel syndrome (ultrasonic swelling of the median nerve, increased palmar bowing of the flexor retinaculum). The electromyelogram confirmed this suspicion.

Sixteen patients experienced tenderness at compression with the transducer along tendons without ultrasonic abnormalities. As repetitive strain injury was suspected, these patients were treated conservatively with splinting or immobilisation in a cast (six weeks) and antiinflammatory drugs.

All 22 patients with probable intra-articular pathology and the remaining five patients without ultrasonic abnormalities underwent a bone scan. Six patients showed diffuse increased uptake compatible with synovitis. In four cases an ultrasound guided synovial biopsy confirmed the diagnosis. As the other two patients developed arthritis of one of the finger joints during follow up, RA was believed to be the cause of the synovitis. Five patients showed markedly increased uptake in the wrist (fig 6). Additional spot films, CT and MRI demonstrated pisotriquetral osteoarthritis, a fracture of hook of the hamate, Kienbock's disease in two cases and carpal boss. In three cases mildly increased uptake was indicative of soft tissue injury. The remaining 13 patients had a negative scan.

Arthrography under fluoroscopy of two of the patients with a positive bone scan and nine patients (six patients with and three patients without "clicking" of the wrist) with a negative bone scan was performed. Ligamentous disruption (one scapholunate articulation, one triquetrolunate articulation) and associated instability patterns were diagnosed for two patients.

Tears of the TFCC were demonstrated in three other patients (fig 5). Two teenage patients had dyssynchronous carpal motion, diagnosed by fluoroscopy without arthrographically documented ligament tears.

Arthroscopy was performed in seven cases: five patients with suspected tears after arthrography, one with clicking of the wrist but 


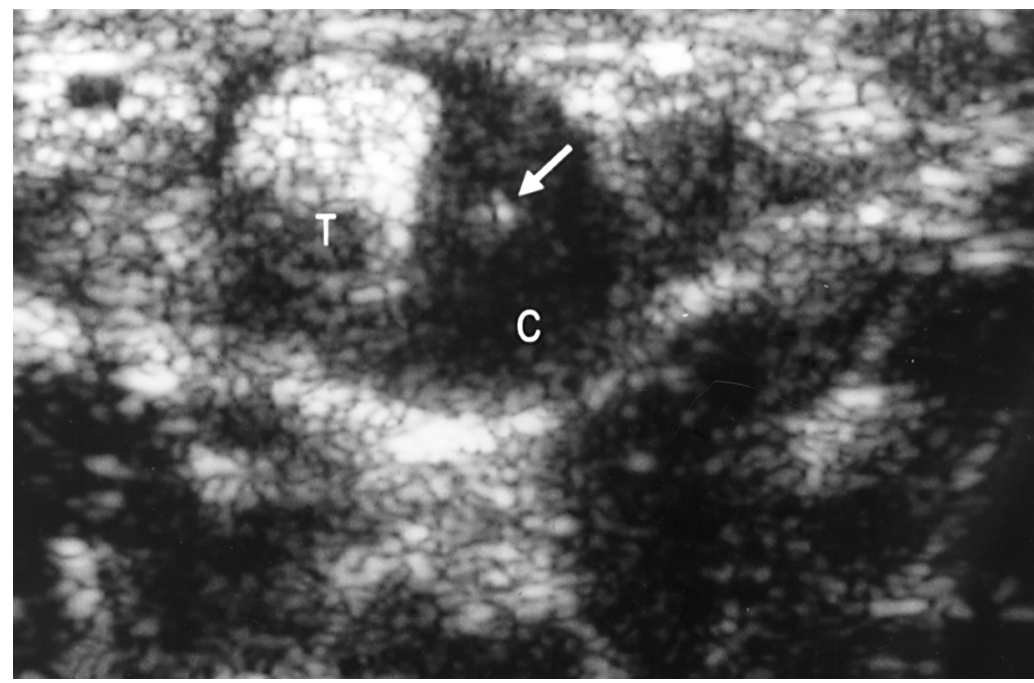

Figure 5 Transverse ultrasound scan of flexor tenosynovitis. The thorn (arrow) can be seen as a hyperechoic stripe in a hypoechoic cavity (C). T: tendon.

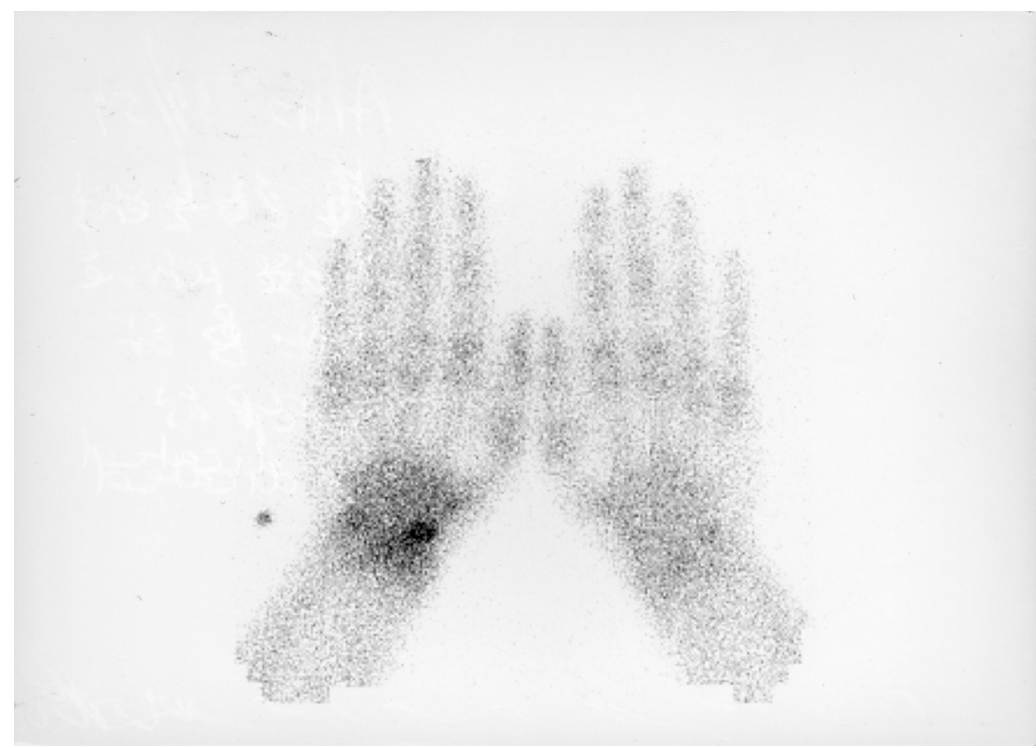

Figure 6 Bone scintigram with markedly increased uptake in the region of the scaphoid.

normal diagnostic studies and one with nondiagnostic calcifications on plain radiographs. Rupture of an interosseous ligament was diagnosed arthroscopically in five cases; in all cases the diagnosis had already been indicated by arthrography. The patient with normal diagnostic studies was found to have a scapholunate tear. The calcified tumours were attributable to osteochondromatosis (fig 7).

A definite diagnosis could be established in $59 \%$ ( 49 of 84 ) of the cases by careful history taking, extensive physical examination, plain radiographs, ultrasound examination and bone scintigraphy (fig 2). In $19 \%$ of the cases (16 of 84) a probable diagnosis was made, resulting in a total figure of $78 \%$ (65 of 84 ). Additional imaging studies (arthrography, MRI and CT) increased the definite diagnoses to $70 \%$ (59 of 84$)$.

\section{Conclusion}

As the wrist can be affected by a multitude of local and general disorders it is often difficult to make an accurate diagnosis. In the literature wrist pain is generally subdivided into pain of

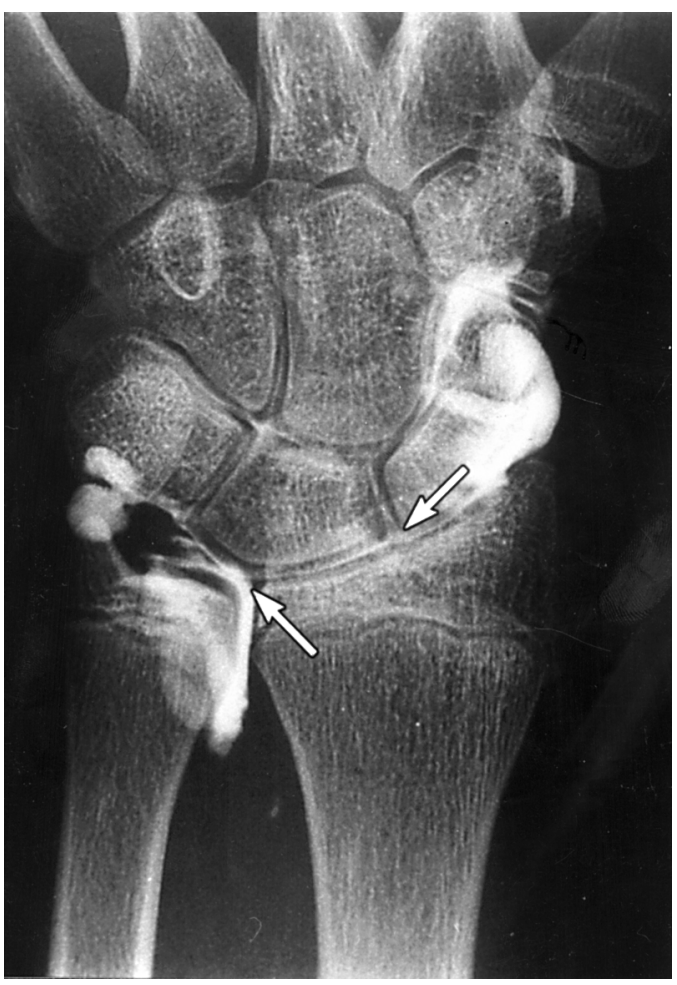

Figure 7 Arthrogram of the radiocarpal joint showing leakage of contrast through the scapholunate ligament and triangular fibrocartilage (arrows) into the mid-carpal and distal radioulnar joints.

traumatic and pain of non-traumatic origin. ${ }^{1478}$ Nevertheless trauma may go unnoticed for some time or the patient may not consider the incident to be relevant to the wrist pain. ${ }^{45}$ Several complicated algorithms have been proposed for the assessment of chronic wrist pain, adding another level of complexity to the evaluation of this already difficult diagnostic problem. ${ }^{149}$ The development of our algorithm was prompted by the need for a clearly defined and easily applied method for identifying the more common causes of wrist pain without having to resort to specialised procedures such as arthroscopy. Our classification of chronic wrist pain as pain of intraarticular or extra-articular origin appeared to be pragmatic and helpful in the examination of chronic wrist pain. By means of this classification a clinical algorithm was developed to establish a diagnosis.

The algorithm proved easy to use; by means of careful history taking, thorough physical examination and simple imaging techniques (plain radiographs, ultrasonography and scintigraphy) a diagnosis was made in $78 \%$ of the cases.

Although this algorithm seems promising for establishing a diagnosis in chronic wrist pain following it will be dependent of the imaging techniques available in each rheumatology unit.

We believe that application of the algorithm described in this paper will vastly increase the rheumatologist's ability to diagnose and treat chronic wrist disorders. This algorithm is by no means exhaustive or complete. Instead it should be viewed as a guide to assist the physician in his management of this difficult problem. 
1 Chidgey LK. Chronic wrist pain. Orthop Clin North Am 1992;23:49-64

2 Taleisnik J. The anatomy of the wrist. In: Taleisnik J, ed. The wrist. New York: Churchill Livingstone, 1985:1-39.

3 Terill RQ. Use of arthroscopy in the evaluation and treatment of chronic wrist pain. Hand Clin 1994;10:593603.

4 Brown DE, Lichtman DM. The evaluation of chronic wrist pain. Orthop Clin North Am 1984;15:183-92.

5 Kasdan ML, Millender LH. Occupational soft-tissue and tendon disorders. Orthop Clinic North Am 1996;27:795803 .

6 McCune WJ. Monoarticular arthritis. In: Kelly WN, Harris ED, eds. Textbook of rheumatology. Philadelphia: WB Saunders Company, 1993:368-79.

7 Taleisnik J. Pain on the ulnar side of the wrist. Hand Clin 1987;3:51-68

8 Taleisnik J. Classification of carpal instability. In: Taleisnik J, ed. The wrist. New York: Churchill Livingstone, 1985:22938.

Linn RM, Gilula LA. Imaging the symptomatic wrist. Orthop Clin North Am 1990;21:515-43.

10 Vugt van RM, Dalen van A, Bijlsma JWJ. Ultrasound guided synovial biopsy of the wrist. Scand J Rheumatol 1997;26: 212-14.

11 Pin PG, Semenkovich JW, Young VL. Role of radionuclide imaging in the evaluation of wrist pain. J Hand Surg 1988; 13A:810-14

12 Hankin FM, White SJ, Braunstein EM. Dynamic radiographic evaluation of obscure wrist pain in the teenage
patient. J Hand Surg 1986;11a:805-9.

patient. J Hand Surg 1986,11a:805-9.

zram. J Hand Surg 1988:13a:803-9. gram. J Hand Surg 1988;13a:803-9.

wrist pain: evaluation with high-resolution MR imaging. Radiology 1989;173:723-9.

15 Schreibman KL, Freeland A, Gilula LA, Yin Y. Imaging of the hand and wrist. Orthop Clin North Am 1997;28:53782.

16 Whipple TL, Marotta JJ, Powell JH. Techniques of wrist arthroscopy. Arthroscopy 1986;2:244-52.

17 Roth JH, Haddad RG. Radiological arthroscopy and arthrography in the diagnosis of ulnar wrist pain. Arthroscopy $1986 ; 2: 234-43$.
18 Fitz Randolph RL. Radiographic and orthopedic evaluation of wrist trauma. Curr Probl Diagn Radiol 1991;2:7-44.

19 Pederzini L. Evaluation of the triangular fibrocartilage complex tears by arthroscopy, arthrography and magnetic resonance imaging. Arthroscopy 1992;8:198-203.

20 Weiss AP, Sachar K, Glowacki KA. Arthroscopic debridement alone for intercarpal ligament tears. J Hand Surg Am 1997;22:344-9.

21 Koski JM. Ultrasonography in detection of effusion in the radiocarpal and midcarpal joints. Scand J Rheumatol 1992;21:79-81.

22 Wilson RL. Rheumatoid arthritis of the hand. Orthop Clin North Am 1986;17:313-43.

23 Belsky MR, Feldon P, Millender LH. Hand involvement in psoriatic arthritis. J Hand Surg 1982;7:203-4

24 Bijlsma JWJ, Breedveld FC, Dequeker J, Linden S van der, Putte LBA van de, Ryswgu MH van. In: Leerboek Reumatologie. Bohn Stafleu VanLoghum, 1992:210-23.

25 Hausman MR, Lisser SP. Hand infections. Orthop Clin North Am 1992;23:171-86.

26 Viegas SF. Atypical causes of hand pain. Am Fam Physician 1987;35:167-72.

27 Watson HK, Brenner LH. Degenerative disorders of the wrist. J Hand Surg 1985;10a:1002-6.

28 Diao E, Moy OJ. Common tumours. Orthop Clin North Am 1992;23:187-96.

29 Gunther SF. Dorsal wrist pain and the occult scapholunate ganglion. J Hand Surg 1985;10a:697-703.

30 Tham S. Intraosseous ganglion cyst of the lunate: diagnosis and management. J Hand Surg 1992;17a:429-32.

31 Resnik CS. Incomplete carpal coalition. Am J Radiol 1986; 147:301-4.

32 Gunther SF. The carpometacarpal joints. Orthop Clin North Am 1984;15:259-78.

33 Weinstein SM. Nerve problems and compartment syndromes in the hand. Clin Sports Med 1992;11:161-88.

34 Carr D, Davis P. Distal posterior interosseous nerve syndrome. J Hand Surg 1985;10a:873-8.

35 Elliot BG. Finkelstein's test; a descriptive error that can produce a false positive. J Hand Surg 1992;17b:481-2.

36 Thorson E, Szabo RM. Common tendinitis problems in the hand and forearm. Orthop Clin North Am 1992;23:65-74. 